Lars Grüter*, Richard Nauber und Jürgen Czarske

\title{
Non-invasive aberration correction and adaptive ultrasound imaging with a time reversal virtual array
}

\author{
Nichtinvasive Aberrationskorrektur und adaptive Ultraschallbildgebung mit einem mit der \\ Zeitumkehrtechnik kalibrierten virtuellen Array
}

DOI 10.1515/teme.2021.0062

Zusammenfassung: Starke akustische Aberrationen, wie sie durch Multimode-Wellenleiter oder den menschlichen Schädel verursacht werden, können die Qualität einer effektiven ultraschallbasierten Bildgebung erheblich reduzieren oder diese verhindern. Wir stellen eine neuartige, nichtinvasive Kalibrierungsmethode vor, die zwei unabhängige akustische Zugänge und ein mithilfe der Zeitumkehrtechnik kalibriertes virtuelles Array (TRVA) zur adaptiven Wellenfrontkorrektur nutzt. Die numerische Charakterisierung und ein Modellexperiment mit dieser Methode zeigen eine Verbesserung der lateralen Auflösung und des Spitze-zu-Hintergrund-Verhältnisses um bis zu $35 \%$ bzw. $10 \%$.

Schlüsselwörter: Zeitumkehr, Systemidentifikation, Bildgebung, Ultraschall.

Abstract: Strong acoustical aberrations such as induced by multi-mode waveguides or the human skull can significantly reduce the quality of or prevent effective ultrasoundbased imaging. We propose a novel non-invasive calibration method that utilizes two independent acoustical accesses and a time reversal virtual array (TRVA) for adaptive wavefront correction. Numerical characterization and a model experiment utilizing this method show an improvement of the lateral resolution and the peak to background ratio by up to $35 \%$ and $10 \%$ respectively.

Keywords: Time reversal, system identification, imaging, ultrasound.

\footnotetext{
*Korrespondenzautor: Lars Grüter, Lehrstuhl für Mess- und Sensorsystemtechnik, Technische Universität Dresden, Helmholtzstraße 18, 01069 Dresden, E-Mail: lars.grueter@tu-dresden.de

Richard Nauber, Jürgen Czarske, Lehrstuhl für Mess- und Sensorsystemtechnik, Technische Universität Dresden, HelmholtzstraBe 18, 01069 Dresden
}

\section{Introduction}

Strokes are one of the most frequent causes of death [5]. The prompt diagnosis and distinction between the ischemic and hemorrhagic subtype is extremely critical to a successful therapy and avoidance of long-term disabilities in patients. Mobile transcranial ultrasound imaging, available in the ambulance, could drastically reduce the time to diagnosis compared to hospital-based x-ray-based imaging. However non-invasive imaging of the brain with ultrasound is obstructed by the aberrating skull bone which significantly degrades the image quality [2]. It has been shown that a time reversal virtual array (TRVA) allows adaptive wavefront correction and planar ultrasound imaging of liquid metals through a aberrating media such as a multi-mode waveguide [3]. This requires system identification by an acoustical point source or reflector behind the aberrator and is therefore invasive. Here we propose a new non-invasive method to calibrate a remote time reversal virtual array (RTRVA) utilizing a second acoustical access. This approach could compensate aberrations by a multimode waveguide or the human skull and improve the image quality in context of transcranial imaging.

\section{Method}

A TRVA is calibrated by identifying the system's impulse response between the TRVA and an ultrasound array. This is achieved by exciting a sound wave, ideally a Dirac delta function, at the position of each virtual element which propagates through an aberrator, is then received with the ultrasound array (A1) and time reversed [3]. The proposed method excites the required calibration signal in a non-invasive manner with a second ultrasound array (A2) which is placed in opposition to A1 as illustrated in Fig. 1. During the calibration phase A2 is focused 


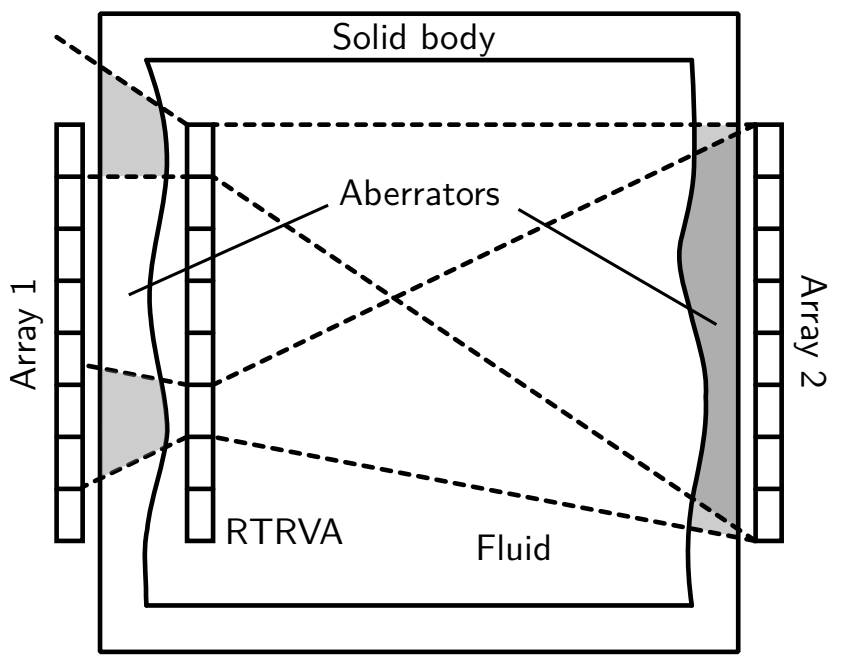

Abb. 1: Focusing on virtual elements during RTRVA calibration inside a simplified skull phantom. The shaded areas highlight the traversed parts of the solid body for two different focus points.

with transmit-side beamforming on the position of each virtual element which later form the RTRVA in front of A1. The focused beam converges at the position of the virtual element and thus acts similar to a sound wave originating from there. Based on the time invariant nature of sound propagation in linear media [1], convolving this received pattern with signals received at A1 during Bmode imaging estimates the signal at each element of the RTRVA free of aberrations.

While the arrays A1 and A2 can access the region of interest only through aberrators, different aberrationinducing parts of the solid body are traversed for different focus points during the calibration phase. Aberrations induced between A2 and the RTRVA do not contribute to the compensation when operating the calibrated RTRVA and represent an undesired calibration error inherent to the method. However, due to the relatively large distance between A2 and the RTRVA compared to its aperture, this calibration error is almost constant for all virtual elements. This mainly leads to an unknown positional offset of the RTRVA as shown later on in the numerical characterization in Fig. 2 which does not reduce the image resolution capabilities.

\section{Characterization}

The proposed calibration method was evaluated numerically and experimentally at $2 \mathrm{MHz}$ inside a water-filled $3 \mathrm{D}$ printed resin phantom. The simulated and experimental setup was chosen similarly to Fig. 1 with two opposing

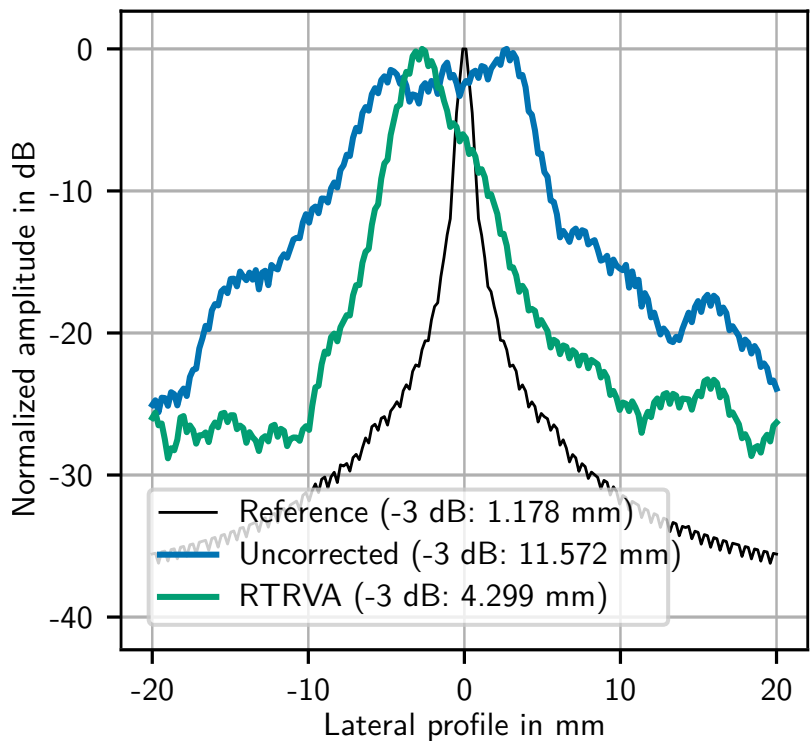

Abb. 2: Simulated lateral intensity profile and beamwidths during transmit-focusing into the phantoms center. The reference profile was computed for a phantom with smooth walls.

phased ultrasound arrays, each with 64 elements. The sound speed of the resin was measured to be $2700 \mathrm{~m} / \mathrm{s}$ and that of water as $1497 \mathrm{~m} / \mathrm{s}$. During the numerical evaluation both the real ultrasound array A1 and the calibrated RTRVA were focused with transmit-side beamforming on the center of the phantom. After calibration of the RTRVA a copper wire with a diameter of $0.5 \mathrm{~mm}$ was placed as a point source between both phased arrays. Utilizing the modular phased array ultrasound system described by Mäder et al. [4] an RTRVA with 64 elements was calibrated as described in section 2 .

Conventional delay-and-sum beamforming with the real array A1 and the RTRVA yielded the simulated and experimental results shown in Fig. 2 and Fig. 3 respectively. The RTRVA can compensate the aberrations induced by the uneven wall thickness and significantly reduces the intensity of artifacts in the lateral direction. For the simulated setup, the RTRVA can reduce the lateral half-width of the transmit-focused beam by $63 \%$ (Fig. 2); for the experimental setup it reduces the lateral half-width of the needle's point-spread function by up to $35 \%$ while increasing the axial half-width by $133 \%$. The latter is likely caused by the calibration signal transmitted by the opposing array A2 which is only an approximation of a Dirac delta function and therefore leads to a temporal widening of the estimated impulse response. As a consequence this extends to the axial beam width during focusing and the axial resolution. 

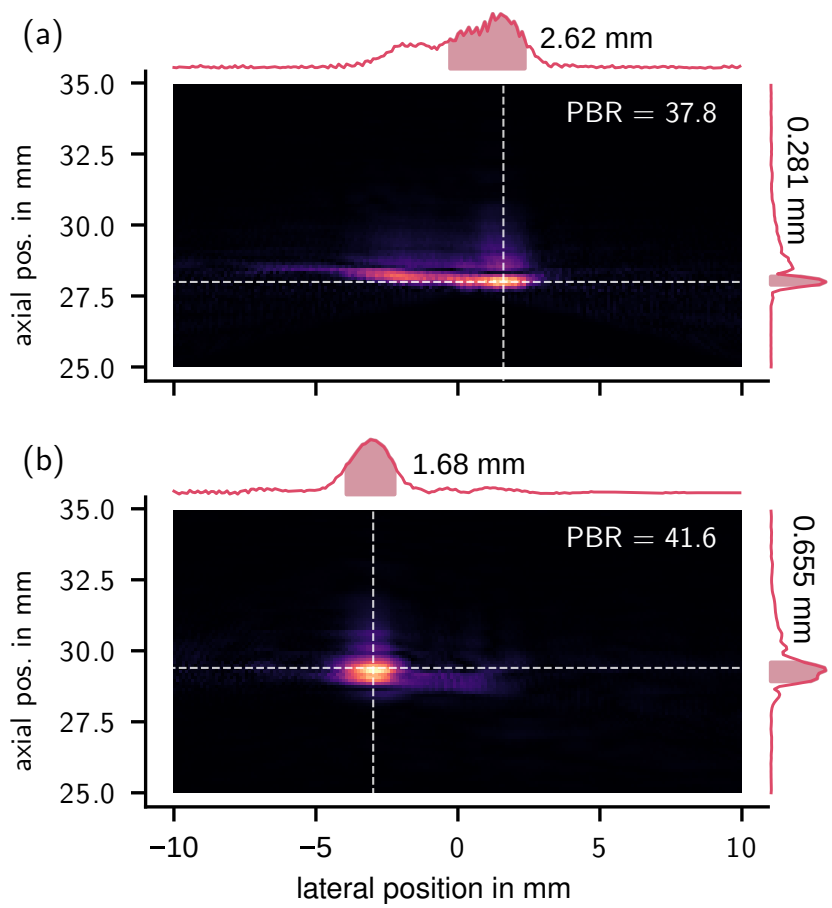

Abb. 3: Resolution and half-widths of a sub-wave-length point source (a) without correction and (b) with a RTRVA inside a simplified skull phantom.

\section{Conclusion and outlook}

The experimental characterization has shown that the proposed novel calibration method improves the signal quality, in case of the peak to background ratio by up to $10 \%$. In the future, the method can be applied to a more accurate skull phantom to examine the effects on image quality in detail.

\section{Literatur}

[1] M. Fink. Time reversal of ultrasonic fields: Part i-basic principles: leee transactions on ultrasonics, ferroelectrics and frequency control, 1992.

[2] F. J. Fry und J. E. Barger. Acoustical properties of the human skull. The Journal of the Acoustical Society of America, 63(5): 1576-1590, 1978.

[3] M. Kalibatas, R. Nauber, C. Kupsch und J. Czarske. Flow field imaging with ultrasonic guided waves for exploring metallic melts. IEEE transactions on ultrasonics, ferroelectrics, and frequency control, 65(1):112-119, 2017.

[4] K. Mäder, R. Nauber, V. Galindo, H. Beyer, L. Büttner, S. Eckert und J. Czarske. Modular phased array ultrasound velocimeter for $2 \mathrm{~d}-2 \mathrm{c}$ flow mapping in liquid metals. IEEE Transactions on Ultrasonics, Ferroelectrics and Frequency Control, 64(9):1327-1335, 2017.
[5] H. Wang, M. Naghavi, C. Allen, R. Barber, Z. Bhutta, A. Carter et al. A systematic analysis for the global burden of disease study 2015. Lancet, 388(10053):1459-1544, 2016. 